\title{
DISTRIBUTED MIN-MAX OPTIMIZATION IN NETWORKS
}

\author{
Kunal Srivastava, Angelia Nedić, and Dušan Stipanović ${ }^{\dagger}$ \\ Industrial and Enterprise System Engineering Department \\ University of Illinois, Urbana-Champaign, USA \\ \{kkunal2,angelia,dusan\}@illinois.edu
}

\begin{abstract}
We consider a setup where we are given a network of agents with their local objective functions which are coupled through a common decision variable. We provide a distributed stochastic gradient algorithm for the agents to compute an optimal decision variable that minimizes the worst case loss incurred by any agent. We establish almost sure convergence of the agent's estimates to a common optimal point. We demonstrate the use of our algorithm to a problem of min-max fair power allocation in a cellular network.
\end{abstract}

Index Terms - Distributed network optimization, minmax problem, subgradient algorithm, stochastic approximation.

\section{INTRODUCTION}

The area of distributed decision making and control has a long history. However, with the proliferation of low cost sensors and microprocessors, this field has seen a resurgence in interest. A canonical problem which has led this resurgence is the problem of reaching consensus on a set of local decision variables in a network of agents [1]. Algorithms for achieving consensus employ local averaging of variables in the network which is shown to be robust to changing graph topology and noisy communication links [2]. These algorithms have led to new distributed algorithms for parameter estimation [3] and distributed optimization [4]. Recent work in consensus based distributed optimization has focused on the problem when the objective is to minimize a sum of convex functions which are local to the agents. Such problems arise in a wide variety of areas from distributed machine learning to resource allocation in a network. The novelty of this paper is the consideration of a min-max criterion for optimization. We are interested in finding the optimal decision variable which minimizes the worst case loss incurred to any agent. This is based on the idea of min-max fairness in a resource allocation problem [5]. We provide a distributed gradient based scheme which leverages ideas from consensus based algorithms to achieve this objective. We allow the presence of stochastic errors in the gra-

\footnotetext{
$\dagger$ The research has been supported by the National Science Foundation under Career grant CMMI 07-42538.
}

dient. This generalizes our formulation to include stochastic optimization problems. Our main result proves the asymptotic convergence of the algorithm to the optimal point with probability one. We also illustrate the applicability of the algorithm in achieving a min-max fair allocation of power in a cellular network.

The rest of the paper is organized as follows. In the next subsection, we introduce our basic notation. In Section 2, we provide a mathematical formulation of the problem of our interest. We discuss some technical results from optimization theory, and we present our algorithm and assumptions on the problem. In Section 3, we provide some preliminary results that we use in Section 4, where we establish the convergence analysis of the proposed algorithm. Finally, in Section 5, we provide simulation results for a power allocation problem in a cellular network.

Notation. All vectors are viewed as columns. The set of real numbers is denoted by $\mathbb{R}$. The $j^{t h}$ component of a vector $x$ is denoted by $x_{j}$. For an $m \times m$ matrix $A$, we use $A_{i j}$ or $[A]_{i j}$ to denote its entry in the $i^{\text {th }}$ row and $j^{\text {th }}$ column. Given a closed convex set $X \subset \mathbb{R}^{n}$, the projection operator $P_{X}[\cdot]$ maps a vector $x \in \mathbb{R}^{n}$ to the closest point to $x$ in the set $X$ under the Euclidean norm. Given a logical statement $p(x)$ predicated on a variable $x$, we use $\mathbf{1}_{\{p(x)\}}$ to denote the function that takes value 1 when $p(x)$ is true and value 0 when $p(x)$ is false.

\section{PROBLEM FORMULATION AND ALGORITHM}

We consider a setup where we are given a set of $m$ agents, which can be viewed as the node set $V=\{1, \ldots, m\}$, and we use the terms node and agent interchangeably. We assume that the time is discrete and use $k=0,1, \ldots$ to denote the time instances. The agents communicate with each other over a time-varying communication network. At any time $k$, the communications among the agents are represented by a directed graph $G(k)=(V, E(k))$ with an edge-set $E(k)$ that has a link $(i, j) \in E(k)$ if and only if agent $i$ receives information from agent $j$ at time $k$.

We consider solving a distributed multi-agent optimization problem subject to local agent communications, where 
the agents want to cooperatively solve the following problem:

$$
\min _{x \in X} \max _{i \in V} f_{i}(x),
$$

where each $f_{i}: \mathbb{R}^{n} \rightarrow \mathbb{R}$ is a convex function, representing a local objective function known only by agent $i$. The set $X \subseteq \mathbb{R}^{n}$ is a closed and convex set known by all agents. The goal is to develop a distributed algorithm for solving the constrained optimization problem in (1). By distributed we mean that the algorithm is executed locally by each agent with a limited information exchange with the agent's one-hop neighbors in the communication graphs $G(k)$. The agents can exchange their iterates that are locally generated by an algorithm. We are interested in the case when the agents' objective functions $f_{i}$ are not necessarily differentiable. In many applications, the local objective functions $f_{i}$ can take the form of the following stochastic optimization:

$$
f_{i}(x)=\mathbb{E}_{\omega_{i}}\left[F_{i}\left(x, \omega_{i}\right)\right]+\Omega(x),
$$

where the expectation is taken with respect to the distribution of the random variable $\omega_{i}$, and the term $\Omega(x)$ is a regularization function. A lot of interest in signal processing has been generated towards the use of the $l_{1}$-norm as the regularization term in order to ensure a sparsity of the solution [6]. Since the $l_{1}$-penalty is non-differentiable, our algorithm which doesn't require the objective function to be differentiable is suitable for this problem. It is well known that the stochastic optimization problems of the form above can be dealt with by using first-order stochastic gradient methods [7-9].

\subsection{Min-Max Problem Reformulation}

The min-max-problem in (1) is a convex problem, as the function $f(x)=\max _{i \in V} f_{i}(x)$ is convex since point-wise maximum of convex functions preserves convexity [10] (Proposition 1.2.4, page 30). However, the form of the min-max problem is not suitable for distributed optimization over the agent network. In our approach, we find it useful to use an epigraph representation of the min-max problem in (1). In particular, we let $\eta \in \mathbb{R}$ and re-cast problem (1) in an equivalent form:

$$
\begin{array}{ll}
\text { minimize } & \eta \\
\text { subject to } & f_{i}(x) \leq \eta \quad \forall x \in X, \eta \in \mathbb{R}, \text { and } i \in V .
\end{array}
$$

In this formulation, the decision variables are $x$ and $\eta$. We assume throughout the paper that the optimal value of the problem is finite and denote this value by $\eta^{*}$. An optimal decision $x^{*} \in X$ for the penalized problem satisfies $f_{i}\left(x^{*}\right) \leq \eta^{*}$ for all $i$. We denote the set of optimal decisions by $X^{*}$. Note that $X^{*}$ is the optimal set for the original min-max problem (1), while $\eta^{*}$ is its optimal value.

We further transform the problem in (3) by penalizing the constraints to obtain the following problem:

$$
\min _{x \in X, \eta \in \mathbb{R}} \eta+\sum_{i=1}^{m} r_{i} g_{i}(x, \eta),
$$

where $g_{i}(x, \eta)=\max \left\{f_{i}(x)-\eta, 0\right\}$ is a penalty function and $r_{i}>0$ is a penalty parameter for violating the constraint $f_{i}(x) \leq \eta$. This problem is suitable for our development of a distributed algorithm, as its objective can be written as $\sum_{i=1}^{m}\left(\eta / m+r_{i} g_{i}(x, \eta)\right)$ and the $i$ th summand can be viewed as an objective function of agent $i$.

Under certain conditions on problem (3) and the penalty parameters $r_{i}$, the solutions of the penalized problem (4) are also the solutions of the constrained problem (3). We next discuss these conditions and show that they are satisfied. The problem of using penalty functions to recover an exact solution of the constrained problem was dealt in [11]. There, a general constrained convex optimization problem was considered, and necessary and sufficient conditions were derived for its equivalence to a penalized problem. The result in [11], when specialized to our problem (3), implies that a solution of the penalized problem (4) is also a solution of the constrained problem (3) if and only if $r_{i}$ is strictly greater than an optimal dual variable corresponding to the $i^{\text {th }}$ constraint in (3). To make this more apparent, let us consider a dual problem to (3), by introducing its Lagrangian function, defined as:

$$
L(x, \eta, \mu)=\eta+\sum_{i=1}^{m} \mu_{i}\left(f_{i}(x)-\eta\right),
$$

where $\mu=\left(\mu_{1}, \ldots, \mu_{m}\right)^{\prime}$ is the vector of dual variables satisfying $\mu_{i} \geq 0$ for all $i \in V$. The dual problem is

$$
\max _{\mu \geq 0} q(\mu) \quad \text { with } \quad q(\mu)=\inf _{x \in X, \eta \in \mathbb{R}} L(x, \eta, \mu) .
$$

It can be verified that the Slater condition is satisfied for problem (3) and, hence, there is no duality gap between the primal problem (3) and its dual (6). Furthermore, the set of dual optimal solutions is nonempty and bounded. The bound for the dual optimal variables can be found by rewriting the Lagrangian function (5) as $L(x, \eta, \mu)=\left(1-\sum_{i=1}^{m} \mu_{i}\right) \eta+$ $\sum_{i=1}^{m} \mu_{i} f_{i}(x)$. Then, we note that $\inf _{\eta \in \mathbb{R}} L(x, \eta, \mu)=-\infty$ when $\sum_{i} \mu_{i} \neq 1$, implying that $q(\mu)=-\infty$ when $\sum_{i} \mu_{i} \neq$ 1. Thus, the domain of the dual function $q$ is the set of multipliers $\mu \geq 0$ such that $\sum_{i=1}^{m} \mu_{i}=1$, implying that optimal multipliers $\mu_{i}^{*}$ also satisfy

$$
\sum_{i=1}^{m} \mu_{i}^{*}=1
$$

Hence, for any optimal multiplier $\mu^{*}=\left(\mu_{1}^{*}, \ldots, \mu_{m}^{*}\right)$ we have $0 \leq \mu_{i}^{*} \leq 1$ for all $i$. According to [11], when the penalty parameters satisfy $r_{i}>1$, then any solution of the penalized problem (4) is also a solution of the constrained problem (3). In what follows, we focus on solving problem (4).

\subsection{Algorithm}

Here, we present a distributed multi-agent algorithm for solving the penalty reformulation (4) of the min-max problem, 
where a penalty function $g_{i}(x, \eta)$ is associated with an agent $i$ and $r_{i}>1$ for all $i$. Let $x_{k}^{j}$ and $\eta_{k}^{j}$ be the decision variables of agent $j$ at time $k$, which are the agent's estimates of the optimal solution $x^{*}$ and the optimal value $\eta^{*}$ of the problem. Recall that the agents' communications at time $k$ are represented with a graph $G(k)=(V, E(k))$, where $(i, j) \in E(k)$ if agent $i$ receives estimates $x^{j}(k)$ and $\eta^{j}(k)$ from agent $j$. To capture this information exchange, let $N_{i}(k)$ denote the set of neighbors of agent $i$, i.e., $N_{i}(k)=\{j \in V \mid(i, j) \in E(k)\}$. Upon receiving the information from its neighbors, each agent $i$ performs an intermittent adjustment of its estimates as follows:

$$
\left[\begin{array}{c}
\tilde{x}_{k}^{i} \\
\tilde{\eta}_{k}^{i}
\end{array}\right]=\sum_{j \in N_{i}(k)} w_{i j}(k)\left[\begin{array}{c}
x_{k}^{j} \\
\eta_{k}^{j}
\end{array}\right]
$$

where $w_{i j}(k) \geq 0$ are weights that agent $i$ assigns to its neighbors $j \in N_{i}(k)$. For a compact representation of relation (8), let $w_{i j}(k)=0$ for all $j \notin N_{i}(k)$ and introduce a matrix $W_{k}$ with entries $w_{i j}(k)$. Then, the intermittent adjustment in (8) reduces to:

$$
\left[\begin{array}{c}
\tilde{x}_{k}^{i} \\
\tilde{\eta}_{k}^{i}
\end{array}\right]=\sum_{j=1}^{m}\left[W_{k}\right]_{i j}\left[\begin{array}{c}
x_{k}^{j} \\
\eta_{k}^{j}
\end{array}\right]
$$

After the intermittent adjustment, each agent takes a step toward minimizing its own penalty function through a two-step adjustment:

$$
\begin{aligned}
& v_{k}^{i}=\left[\begin{array}{c}
\tilde{x}_{k}^{i} \\
\tilde{\eta}_{k}^{i}
\end{array}\right]-\frac{\alpha_{k}}{m}\left[\begin{array}{c}
0_{n} \\
1
\end{array}\right] \\
& {\left[\begin{array}{c}
x_{k+1}^{i} \\
\eta_{k+1}^{i}
\end{array}\right]=P_{X \times \mathbb{R}}\left[v_{k}^{i}-\alpha_{k} r_{i}\left(\nabla g_{i}\left(v_{k}^{i}\right)+\left[\begin{array}{c}
\epsilon_{k}^{i} \\
0
\end{array}\right]\right)\right]}
\end{aligned}
$$

where $P_{X \times \mathbb{R}}$ is the Euclidean projection on the product set $X \times \mathbb{R}, r_{i}>1$ and $\alpha_{k}>0$ is a step size.

When $v_{k}^{i}$ is computed, only the value $\tilde{\eta}_{k}^{i}$ is updated. The second update involves taking a step along an erroneous subgradient of $\nabla g_{i}\left(v_{k}^{i}\right)$, where $\epsilon_{k}^{i}$ is a subgradient error. Note that agent $i$ objective function $g_{i}(x, \eta)=\max \left\{0, f_{i}(x)-\eta\right\}$ is not differentiable at the point $(x, \eta)$ where $f_{i}(x)-\eta=0$. Then, a subgradient of the function $g_{i}$ at $(x, \eta)$ is given by

$$
\nabla g_{i}(x, \eta)=\left[\begin{array}{c}
\nabla f_{i}(x) \\
-1
\end{array}\right] \mathbf{1}_{\left\{f_{i}(x) \geq \eta\right\}},
$$

where $\nabla f_{i}(x)$ denotes a subgradient of the function $f_{i}$ at $x$. Since each function $f_{i}$ is assumed to be convex over the entire space $\mathbb{R}^{n}$, the subdifferential set $\partial f_{i}(x)$ is nonempty for all $x$ and $i \in V$ (see [10], Proposition 4.2.1). Thus, the function $g_{i}$ also has a nonempty subdifferential set at any point $(x, \eta)$. The first step of computing $v_{k}^{i}$ is crucial. It means that the agents first re-adjust their estimates of optimal value $\eta^{*}$ for a better alignment with their neighbors and, then, the agents proceed with computing new estimates of the optimal decision and the optimal value. This turned to be a crucial detail for the algorithm's convergence.
The initial points $x_{0}^{i} \in X$ and $\eta_{0}^{i}$ may be selected randomly with the distribution independent of any other sources of randomness in the algorithm. The subgradient error $\epsilon_{k}^{i}$ is assumed to be stochastic in order to tackle the general form of the objective function as in (2), where the subgradient $\nabla f_{i}(x)$ is not readily available to us. Here, we adopt a standard approach in stochastic optimization by using an unbiased estimate $\nabla f_{i}(x)+\epsilon_{k}^{i}$ of the subgradient, where $\epsilon_{k}^{i}$ is a zero mean random variable. These assumptions, as well as the assumptions on the weight matrices and the underlying communication graphs are discussed in the following section.

\subsection{Assumptions}

Our assumptions on the network are similar to those in [4]. The assumptions ensure that agent's local variables are properly diffused over the communication networks in time. More discussion on the role of these assumptions can be found in [4].

Assumption 1. For the weight matrices and the communication graphs, we assume the following:

a) (Weights rule) There exists a scalar $0<\gamma<1$ such that $\left[W_{k}\right]_{i i} \geq \gamma$ for all $i$ and $k$, and $\left[W_{k}\right]_{i j} \geq \gamma$ if $\left[W_{k}\right]_{i j}>0$.

b) (Doubly Stochasticity) The matrix $W_{k}$ is doubly stochastic for all $k$, i.e., $\sum_{j=1}^{m}\left[W_{k}\right]_{i j}=1$ and $\sum_{i=1}^{m}\left[W_{k}\right]_{i j}=1$.

c) (Connectedness) There exists an integer $B \geq 1$ such that the graph $\left(V, \cup_{\tau=k B}^{(k+1) B-1} E(\tau)\right)$ is strongly connected for every $k$.

We impose the following assumptions on the subgradients $\nabla f_{i}(x)$ and the errors $\epsilon_{k}^{i}$.

Assumption 2. Let the following hold:

a) The subgradients of each $f_{i}$ are bounded over the set $X$, i.e., there is a scalar $C>0$ such that $\left\|\nabla f_{i}(x)\right\| \leq C$ for all $\nabla f_{i}(x) \in \partial f_{i}(x)$, all $x \in X$ and all $i$.

b) The subgradient errors $\epsilon_{k}^{i}$ when conditioned on the point $x=\tilde{x}_{k}^{i}$ of the subgradient $\nabla f_{i}(x)$ evaluation are zero mean, i.e., $\mathbb{E}\left[\epsilon_{k}^{i} \mid \tilde{x}_{k}^{i}\right]=0$ for all $i$ and $k \geq 0$ with probability 1 .

c) There is a scalar $\nu>0$ such that $\mathbb{E}\left[\left\|\epsilon_{k}^{i}\right\|^{2} \mid \tilde{x}_{k}^{i}\right] \leq \nu^{2}$ for all $i$ and $k \geq 0$ with probability 1 .

In what follows, we will use $F_{k}$ to denote the past iterates of the algorithm (9)-(10), i.e.,

$$
F_{k}=\left\{x_{t}^{i}, \eta_{t}^{i}, i \in V, t=0,1, \ldots, k\right\} \quad \text { for } k \geq 0 .
$$

Note that, given $F_{k}$, the iterates $\tilde{x}_{k}^{i}$ and $\tilde{\eta}_{k}^{i}$ in (9) are deterministic. In view of this, as a consequence of the subgradient 
norm and subgradient error boundedness (Assumptions 2-a and 2-c), it can be seen that with probability 1 ,

$$
\mathbb{E}\left[\left\|\nabla f_{i}(x)+\epsilon_{k}^{i}\right\|^{2} \mid F_{k}\right] \leq(C+\nu)^{2} .
$$

Here, we have used Hölder's inequality, which states that $\mathbb{E}\left[\left|x^{\prime} y\right|\right] \leq \sqrt{\mathbb{E}\left[\|x\|^{2}\right]} \sqrt{\mathbb{E}\left[\|y\|^{2}\right]}$ for random vectors $x$ and $y$. In view of the expression for $\nabla g_{i}(x, \eta)$, we can further see that with probability 1 for all $i$ and $k \geq 0$,

$$
\mathbb{E}\left[\left\|\nabla g_{i}\left(v_{k}^{i}\right)+\left[\begin{array}{c}
\epsilon_{k}^{i} \\
0
\end{array}\right]\right\|^{2} \mid F_{k}\right] \leq(C+\nu)^{2}+1 .
$$

\section{PRELIMINARY RESULTS}

Our first lemma establishes an important relation between the optimal solutions of the min-max problem formulation in (3) and its penalized counterpart in (4). In the proof of the lemma, we use the saddle point theorem characterizing the optimal solutions of the problem (3) and its dual problem (6), as given for example in [10], Proposition 6.2.4, page 360.

Theorem 1. (Saddle-Point Theorem) The pair $\left(z^{*}, \mu^{*}\right)$ with $z^{*}=\left(x^{*}, \eta^{*}\right) \in X \times \mathbb{R}$ and $\mu^{*} \geq 0$ is a primal-dual optimal solution pair if and only if the following relation holds:

$$
L\left(z^{*}, \mu\right) \leq L\left(z^{*}, \mu^{*}\right) \leq L\left(z, \mu^{*}\right),
$$

for all $z=(x, \eta) \in X \times \mathbb{R}$ and $\mu \geq 0$, i.e., $\left(z^{*}, \mu^{*}\right)$ is a saddle point of the Lagrangian function $L(z, \mu)$.

Now, we state the lemma.

Lemma 1. Let $\eta^{*}=\min _{x \in X} \max _{i} f_{i}(x)$ and $r_{i}>1$ for all $i$. Then, for $g_{i}(x, \eta)=\max \left\{0, f_{i}(x)-\eta\right\}$ we have

$$
\sum_{i=1}^{m} r_{i} g_{i}(x, \eta)+\eta \geq \eta^{*} \quad \text { for all } x \in X \text { and } \eta \in \mathbb{R} .
$$

Furthermore, equality holds in the above expression if and only if $\eta=\eta^{*}$ and $x=x^{*}$ for an optimal solution $x^{*}$ of the problem $\min _{x \in X} \max _{i} f_{i}(x)$.

Proof. Consider the definition of the Lagrangian in (5). Then, $\eta^{*}=L\left(z^{*}, \mu^{*}\right)$. Now, for the given $x \in X$ and $\eta$, let us define the dual variables $\mu_{i}$ such that $\mu_{i}=r_{i}$ if $f_{i}(x)-\eta \geq 0$, and $\mu_{i}=0$ if $f_{i}(x)-\eta<0$. Then, we have

$\sum_{i=1}^{m} r_{i} \max \left\{f_{i}(x)-\eta, 0\right\}+\eta=\sum_{i=}^{m} \mu_{i}\left(f_{i}(x)-\eta\right)+\eta=L(z, \mu)$.

We need to prove that $L(z, \mu)-L\left(z^{*}, \mu^{*}\right) \geq 0$. From Theo- rem 1, we have $-L\left(z^{*}, \mu^{*}\right) \geq-L\left(z, \mu^{*}\right)$, implying

$$
\begin{aligned}
& L(z, \mu)-L\left(z^{*}, \mu^{*}\right) \geq L(z, \mu)-L\left(z, \mu^{*}\right) \\
&= \sum_{i=1}^{m}\left(\mu_{i}-\mu_{i}^{*}\right)\left(f_{i}(x)-\eta\right) \\
&= \sum_{i=1}^{m}\left(r_{i}-\mu_{i}^{*}\right) \mathbf{1}_{\left\{f_{i}(x) \geq \eta\right\}}\left(f_{i}(x)-\eta\right) \\
&-\sum_{i=1}^{m} \mu_{i}^{*} \mathbf{1}_{\left\{f_{i}(x)<\eta\right\}}\left(f_{i}(x)-\eta\right) \\
& \geq 0,
\end{aligned}
$$

where we use $0 \leq \mu_{i}^{*} \leq 1$ (see (7)) and $r_{i}>1$ for all $i$.

We now prove the second assertion. By the definition of min-max solution we have $f_{i}\left(x^{*}\right) \leq \eta^{*}$ for all $i$, so that $\sum_{i=1}^{m} r_{i} \max \left\{f_{i}\left(x^{*}\right)-\eta^{*}, 0\right\}+\eta^{*}=\eta^{*}$. Thus, we just need to prove the "only if" part. For this, let us assume that for some $x \in X$ and $\eta$ we have

$$
\sum_{i=1}^{m} r_{i} \max \left\{f_{i}(x)-\eta, 0\right\}+\eta=\eta^{*} .
$$

Since $\sum_{i=1}^{m} r_{i} \max \left\{f_{i}(x)-\eta, 0\right\} \geq 0$, it follows $\eta \leq \eta^{*}$. Let us assume that $\eta<\eta^{*}$. Then for the equality to hold, we must have $f_{j}(x)>\eta$ for some $j$. Thus, $f_{i^{*}}(x)>\eta$ for $i^{*}=\operatorname{argmax}_{i} f_{i}(x)$. By $\eta^{*}=\min _{x \in X} \max _{i} f_{i}(x)$ we have $f_{i^{*}}(x) \geq \eta^{*}$ implying $f_{i^{*}}(x)-\eta \geq \eta^{*}-\eta>0$. Since $r_{i^{*}}>1$, it follows that $r_{i^{*}}\left(f_{i^{*}}(x)-\eta\right)>\eta^{*}-\eta$. Therefore, $\sum_{i=1}^{m} r_{i} \max \left\{f_{i}(x)-\eta, 0\right\}+\eta>\eta^{*}$, which is a contradiction; so we must have $\eta=\eta^{*}$ in (13). This however yields $f_{i}(x) \leq \eta^{*}$ for all $i$, thus showing that $x$ is the min-max solution.

We now show some preliminary relations for the iterates of the algorithm. We start with a result related to the instantaneous averages of the iterates $x_{k}^{i}$ and $\eta_{k}^{i}$ over $i \in V$, defined by

$$
\hat{x}_{k}=\frac{1}{m} \sum_{i=1}^{m} x_{k}^{i}, \quad \hat{\eta}_{k}=\frac{1}{m} \sum_{i=1}^{m} \eta_{k}^{i} \quad \text { for all } k \geq 0 .
$$

The result is a simple extension of Theorem 6.1 in [4].

Lemma 2. Let Assumptions 1 and 2 hold, and let the step sizes satisfy $\sum_{k=0}^{\infty} \alpha_{k}^{2}<\infty$. Then, for the iterates of the algorithm (9)-(10) we have with probability 1 for all $j \in V$,

$$
\sum_{k=1}^{\infty} \alpha_{k}\left\|\hat{x}_{k}-x_{k}^{j}\right\|<\infty, \quad \sum_{k=1}^{\infty} \alpha_{k}\left|\hat{\eta}_{k}-\eta_{k}^{j}\right|<\infty .
$$

We next state a result for later use in the convergence proof for the algorithm.

Lemma 3. ([12], page 50) Let $\left\{X_{t}\right\},\left\{Y_{t}\right\},\left\{Z_{t}\right\}$ and $\left\{a_{t}\right\}$ be sequences of random variables, and let $\mathcal{F}_{t}, t=0,1,2, \ldots$, be a filtration such that $\mathcal{F}_{t} \subseteq \mathcal{F}_{t+1}$ for $t \geq 0$. Suppose that: 
a) The random variables $Y_{t}, X_{t}, Z_{t}$ and $a_{t}$ are nonnegative, and are adapted to the filtration $\mathcal{F}_{t}$.

b) For each $t$, we have almost surely

$$
\mathbb{E}\left[Y_{t+1} \mid \mathcal{F}_{t}\right] \leq\left(1+a_{t}\right) Y_{t}-X_{t}+Z_{t} .
$$

c) $\sum_{t=0}^{\infty} Z_{t}<\infty$ and $\sum_{t=0}^{\infty} a_{t}<\infty$ almost surely.

Then, almost surely, $\sum_{t=0}^{\infty} X_{t}<\infty$ and the sequence $Y_{t}$ converges to a nonnegative random variable $Y$.

\section{ANALYSIS OF THE ALGORITHM}

We use techniques from Lyapunov analysis to prove convergence of our algorithm. We also let

$$
z_{k}^{i}=\left[\begin{array}{c}
x_{k}^{i} \\
\eta_{k}^{i}
\end{array}\right], \quad \tilde{z}_{k}^{i}=\left[\begin{array}{c}
\tilde{x}_{k}^{i} \\
\tilde{\eta}_{k}^{i}
\end{array}\right], \quad \hat{z}_{k}=\left[\begin{array}{c}
\hat{x}_{k} \\
\hat{\eta}_{k}
\end{array}\right] .
$$

The following lemma establishes a descent type condition for the iterations of the algorithm.

Lemma 4. Let Assumptions 1 and 2 hold. Then, for algorithm (9)-(10) we have with probability 1 for any $z^{*} \in$ $X^{*} \times\left\{\eta^{*}\right\}$ and any $k \geq 0$,

$$
\begin{aligned}
& \sum_{i=1}^{m} \mathbb{E}\left[\left\|z_{k+1}^{i}-z^{*}\right\|^{2} \mid F_{k}\right] \leq \sum_{j=1}^{m}\left\|z_{k}^{j}-z^{*}\right\|^{2}+\alpha_{k}^{2} C_{2} \\
& -2 \alpha_{k}\left(\sum_{i=1}^{m} r_{i} g_{i}\left(\hat{z}_{k}\right)+\hat{\eta}_{k}-\eta^{*}\right) \\
& +2 \alpha_{k} \bar{r}\left(C \sum_{j=1}^{m}\left\|\hat{x}_{k}-x_{k}^{j}\right\|+\sum_{j=1}^{m}\left|\hat{\eta}_{k}-\eta_{k}^{j}\right|\right)+2 \alpha_{k}^{2} \bar{r}
\end{aligned}
$$

where $C_{2}=1 / m+C_{1} \sum_{i=1}^{m} r_{i}^{2}, C_{1}=(C+\nu)^{2}+1$, and $\bar{r}=\max _{i} r_{i}$.

Proof. Consider the algorithm as given in (9)-(10). Then, using the notation in (14) and utilizing the non-expansiveness property of the projection operator [10], Proposition 2.2.1, page 88 , we have for any $z^{*} \in X^{*} \times\left\{\eta^{*}\right\}$, any $k \geq 0$ and $i \in V$,

$$
\begin{aligned}
\left\|z_{k+1}^{i}-z^{*}\right\|^{2} & \leq\left\|v_{k}^{i}-z^{*}\right\|^{2}+r_{i}^{2} \alpha_{k}^{2}\left\|\nabla g_{i}\left(v_{k}^{i}\right)+\left[\begin{array}{c}
\epsilon_{k}^{i} \\
0
\end{array}\right]\right\|^{2} \\
& -2 \alpha_{k} r_{i}\left(\nabla g_{i}\left(v_{k}^{i}\right)+\left[\begin{array}{c}
\epsilon_{k}^{i} \\
0
\end{array}\right]\right)^{\prime}\left(v_{k}^{i}-z^{*}\right) .
\end{aligned}
$$

Taking the expectation conditional on $F_{k}$, and using relation (12) and the zero mean error $\epsilon_{k}^{i}$ assumption (Assumption -b), we obtain

$$
\begin{aligned}
\mathbb{E}\left[\left\|z_{k+1}^{i}-z^{*}\right\|^{2} \mid F_{k}\right] \leq & \left\|v_{k}^{i}-z^{*}\right\|^{2}+r_{i}^{2} \alpha_{k}^{2} C_{1} \\
& -2 \alpha_{k} r_{i} \nabla g_{i}\left(v_{k}^{i}\right)^{\prime}\left(v_{k}^{i}-z^{*}\right),
\end{aligned}
$$

where $C_{1}=(C+\nu)^{2}+1$. From the definition of $v_{k}^{i}$ in (10) we have

$$
\left\|v_{k}^{i}-z^{*}\right\|^{2}=\left\|\tilde{z}_{k}^{i}-z^{*}\right\|^{2}+\frac{\alpha_{k}^{2}}{m^{2}}-2 \frac{\alpha_{k}}{m}\left(\tilde{\eta}_{k}^{i}-\eta^{*}\right) .
$$

By combining the preceding two relations, we obtain for any $z^{*} \in X^{*} \times\left\{\eta^{*}\right\}$, any $k \geq 0$ and $i \in V$,

$$
\begin{aligned}
& \mathbb{E}\left[\left\|z_{k+1}^{i}-z^{*}\right\|^{2} \mid F_{k}\right] \leq\left\|\tilde{z}_{k}^{i}-z^{*}\right\|^{2}+\frac{\alpha_{k}^{2}}{m^{2}}+r_{i}^{2} \alpha_{k}^{2} C_{1} \\
& -2 \alpha_{k} r_{i} \nabla g_{i}\left(v_{k}^{i}\right)^{\prime}\left(v_{k}^{i}-z^{*}\right)-2 \frac{\alpha_{k}}{m}\left(\tilde{\eta}_{k}^{i}-\eta^{*}\right) .
\end{aligned}
$$

From the convexity of $g_{i}$ and the subgradient property we have

$$
\nabla g_{i}\left(v_{k}^{i}\right)^{\prime}\left(v_{k}^{i}-z^{*}\right) \geq g_{i}\left(v_{k}^{i}\right)-g_{i}\left(z^{*}\right)=g_{i}\left(v_{k}^{i}\right),
$$

where the equality follows by $g_{i}\left(z^{*}\right)=\max \left\{0, f_{i}\left(x^{*}\right)-\eta^{*}\right\}$ and relation $f_{i}\left(x^{*}\right)-\eta^{*} \leq 0$ which holds for all $i$ at any optimal point $x^{*}$ of the problem. Therefore, for any $z^{*} \in$ $X^{*} \times\left\{\eta^{*}\right\}$, any $k \geq 0$ and $i \in V$,

$$
\begin{aligned}
& \mathbb{E}\left[\left\|z_{k+1}^{i}-z^{*}\right\|^{2} \mid F_{k}\right] \leq\left\|\tilde{z}_{k}^{i}-z^{*}\right\|^{2}+\frac{\alpha_{k}^{2}}{m^{2}}+r_{i}^{2} \alpha_{k}^{2} C_{1} \\
& -2 \alpha_{k} r_{i} g_{i}\left(v_{k}^{i}\right)-2 \frac{\alpha_{k}}{m}\left(\tilde{\eta}_{k}^{i}-\eta^{*}\right) .
\end{aligned}
$$

Now, by the convexity of the norm and the doubly stochasticity of the weight matrix $W_{k}$ (Assumption 1-b) we have

$$
\begin{aligned}
\sum_{i=1}^{m}\left\|\tilde{z}_{k}^{i}-z^{*}\right\|^{2} & =\sum_{i=1}^{m}\left\|\sum_{j=1}^{m}\left[W_{k}\right]_{i j} z_{k}^{j}-z^{*}\right\|^{2} \\
& \leq \sum_{i=1}^{m} \sum_{j=1}^{m}\left[W_{k}\right]_{i j}\left\|z_{k}^{j}-z^{*}\right\|^{2} \\
& =\sum_{j=1}^{m}\left\|z_{k}^{j}-z^{*}\right\|^{2} .
\end{aligned}
$$

Thus, summing inequalities in (15) over $i \in V$ we obtain for any $z^{*} \in X^{*} \times\left\{\eta^{*}\right\}$ and all $k \geq 0$,

$$
\begin{aligned}
& \sum_{i=1}^{m} \mathbb{E}\left[\left\|z_{k+1}^{i}-z^{*}\right\|^{2} \mid F_{k}\right] \leq \sum_{j=1}^{m}\left\|z_{k}^{j}-z^{*}\right\|^{2}+\alpha_{k}^{2} C_{2} \\
& -2 \alpha_{k} \sum_{i=1}^{m} r_{i} g_{i}\left(v_{k}^{i}\right)-2 \alpha_{k} \frac{1}{m} \sum_{i=1}^{m}\left(\tilde{\eta}_{k}^{i}-\eta^{*}\right),
\end{aligned}
$$

where $C_{2}=\frac{1}{m}+C_{1} \sum_{i=1}^{m} r_{i}^{2}$.

Now, recalling the definition of $\tilde{\eta}_{k}^{i}$ and the doubly stochasticity of the matrix $W_{k}$, we have

$$
\sum_{i=1}^{m} \tilde{\eta}_{k}^{i}=\sum_{i=1}^{m} \sum_{j=1}^{m}\left[W_{k}\right]_{i j} \eta_{k}^{j}=\sum_{j=1}^{m} \eta_{k}^{j}=m \hat{\eta}_{k}
$$


which when substituted in (16) yields

$$
\begin{aligned}
& \sum_{i=1}^{m} \mathbb{E}\left[\left\|z_{k+1}^{i}-z^{*}\right\|^{2} \mid F_{k}\right] \leq \sum_{j=1}^{m}\left\|z_{k}^{j}-z^{*}\right\|^{2}+\alpha_{k}^{2} C_{2} \\
& -2 \alpha_{k} \sum_{i=1}^{m} r_{i} g_{i}\left(v_{k}^{i}\right)-2 \alpha_{k}\left(\hat{\eta}_{k}-\eta^{*}\right) .
\end{aligned}
$$

Next, recalling $\hat{z}_{k}$ from (14), upon adding and subtracting the term $2 \alpha_{k} \sum_{i} r_{i} g_{i}\left(\hat{z}_{k}\right)$ in (17), we get

$$
\begin{aligned}
& \sum_{i=1}^{m} \mathbb{E}\left[\left\|z_{k+1}^{i}-z^{*}\right\|^{2} \mid F_{k}\right] \leq \sum_{j=1}^{m}\left\|z_{k}^{j}-z^{*}\right\|^{2}+\alpha_{k}^{2} C_{2} \\
& -2 \alpha_{k}\left(\sum_{i=1}^{m} r_{i} g_{i}\left(\hat{z}_{k}\right)+\hat{\eta}_{k}-\eta^{*}\right) \\
& +2 \alpha_{k} \sum_{i=1}^{m} r_{i}\left|g_{i}\left(\hat{z}_{k}\right)-g_{i}\left(v_{k}^{i}\right)\right| .
\end{aligned}
$$

Next, consider the term $\sum_{i} r_{i}\left|g_{i}\left(\hat{z}_{k}\right)-g_{i}\left(v_{k}^{i}\right)\right|$. By the definition of $g_{i}$ and relation $|\max \{a, 0\}-\max \{b, 0\}| \leq|a-b|$ valid for any two scalars $a$ and $b$, we have the following:

$$
\begin{aligned}
& \left|g_{i}\left(\hat{z}_{k}\right)-g_{i}\left(v_{k}^{i}\right)\right| \leq\left|f_{i}\left(\hat{x}_{k}\right)-f_{i}\left(\tilde{x}_{k}^{i}\right)-\hat{\eta}_{k}+\tilde{\eta}_{k}^{i}-\alpha_{k} / m\right| \\
& \leq\left|f_{i}\left(\hat{x}_{k}\right)-f_{i}\left(\tilde{x}_{k}^{i}\right)\right|+\left|\hat{\eta}_{k}-\tilde{\eta}_{k}^{i}\right|+\alpha_{k} / m \\
& \leq \sum_{j=1}^{m}\left[W_{k}\right]_{i j}\left[C \| \hat{x}_{k}-x_{k}^{j}||+\left|\hat{\eta}_{k}-\eta_{k}^{j}\right|\right]+\alpha_{k} / m,
\end{aligned}
$$

where in the first inequality we use the definition of $v_{k}^{i}$, while in the last inequality we use the subgradient boundedness assumption for $f_{i}$ and the definition of the variables $\tilde{x}_{k}^{i}$ and $\tilde{\eta}_{k}^{i}$ in (9). Therefore, by using the doubly stochasticity of $W_{k}$, we obtain

$$
\begin{aligned}
& \sum_{i=1}^{m} r_{i}\left|g_{i}\left(\hat{z}_{k}\right)-g_{i}\left(v_{k}^{i}\right)\right| \leq \bar{r} C \sum_{j=1}^{m}\left\|\hat{x}_{k}-x_{k}^{j}\right\| \\
& +\bar{r} \sum_{j=1}^{m}\left|\hat{\eta}_{k}-\eta_{k}^{j}\right|+\bar{r} \alpha_{k},
\end{aligned}
$$

where $\bar{r}=\max _{i} r_{i}$. Upon substituting the bound (19) in (18), we obtain the desired result.

We are now ready to prove our main convergence result. The result essentially states that under suitable conditions on the step size $\alpha_{k}$, all the agents' estimates converge to a common optimal point. Moreover, the agents' estimates of the min-max value also converge to the optimal value of the problem.

Theorem 2. Let Assumptions 1 and 2 hold. Assume that minmax problem (1) has an optimal solution set $X^{*} \subseteq X$. Let the step sizes satisfy $\sum_{k=0}^{\infty} \alpha_{k}=\infty$ and $\sum_{k=0}^{\infty} \alpha_{k}^{2}<\infty$. Then, the agents' iterates $x_{k}^{i}$ and $\eta_{k}^{i}$ generated by algorithm (9)(10) are such that with probability 1: the decision variables $x_{k}^{i}$ converge to a common optimal (random) point $x^{*} \in X^{*}$ for all $i$ and the estimates $\eta_{k}^{i}$ converge to the optimal value $\eta^{*}$ of the min-max problem.

Proof. Our analysis is based on applying the RobbinsSiegmund result from Lemma 3 to the inequality derived in Lemma 4. By our assumption on the step sizes we have $\sum_{k=1}^{\infty} \alpha_{k}^{2}<\infty$, which trivially implies that $\sum_{k=1}^{\infty} 2(\bar{r}+$ $\left.C_{1} / 2\right) \alpha_{k}^{2}<\infty$. Furthermore, by virtue of Lemma 2 we have

$$
\sum_{k=1}^{\infty} 2 \alpha_{k} \bar{r} \sum_{j=1}^{m}\left(C\left\|\hat{x}_{k}-x_{k}^{j}\right\|+\left|\hat{\eta}_{k}-\eta_{k}^{j}\right|\right)<\infty .
$$

In addition, by virtue of Lemma 1 we have that

$$
\sum_{i=1}^{m} r_{i} g_{i}\left(\hat{z}_{k}\right)+\hat{\eta}_{k}-\eta^{*} \geq 0 \quad \text { for all } k \geq 0 .
$$

Thus, we can apply Lemma 3 and infer that with probability 1 , $\sum_{i=1}^{m}\left\|z_{k}^{i}-z^{*}\right\|^{2}$ converges for every $z^{*} \in X^{*} \times\left\{\eta^{*}\right\}$ and

$$
\sum_{k=1}^{\infty} \alpha_{k}\left(\sum_{i=1}^{m} r_{i} g_{i}\left(\hat{z}_{k}\right)+\hat{\eta}_{k}-\eta^{*}\right)<\infty .
$$

Now, since $\sum_{k=1}^{\infty} \alpha_{k}=\infty$, without loss of generality we can use Cantor diagonalization-type argument to select a subsequence such that the following hold with probability 1 ,

$$
\begin{aligned}
& \lim _{\ell \rightarrow \infty}\left(\sum_{i=1}^{m} r_{i} g_{i}\left(\hat{z}_{k_{\ell}}\right)+\hat{\eta}_{k_{\ell}}-\eta^{*}\right)=0, \\
& \lim _{\ell \rightarrow \infty}\left\|\hat{x}_{k_{\ell}}-x_{k_{\ell}}^{j}\right\|=0 \text { and } \lim _{\ell \rightarrow \infty}\left|\hat{\eta}_{k_{\ell}}-\eta_{k_{\ell}}^{j}\right|=0 \forall j .
\end{aligned}
$$

This implies that along a further subsequence, which without loss of generality we can let it be indexed by the same index set $\left\{k_{\ell}, \ell=1,2, \ldots\right\}$, with probability 1 we have $\lim _{\ell \rightarrow \infty} \hat{x}_{k_{\ell}}=x^{\star}$ and $\lim _{\ell \rightarrow \infty} \hat{\eta}_{k \ell}=\eta^{\star}$. Moreover, with probability 1 the limit points satisfy

$$
\sum_{i=1}^{m} r_{i} g_{i}\left(x^{\star}\right)+\eta^{\star}-\eta^{*}=0
$$

From Lemma 1 it follows that $x^{\star}$ is an optimal solution to the min-max problem with probability 1 and $\eta^{\star}=\eta^{*}$. In view of (22), with probability 1 , we further have $x_{k_{\ell}}^{j} \rightarrow x^{\star}$ and $\eta_{k_{\ell}}^{j} \rightarrow \eta^{*}$ for all $j$. Let $z^{\star}=\left(x^{\star}, \eta^{*}\right)$, then we have $\lim _{\ell \rightarrow \infty}\left\|z_{k_{\ell}}^{j}-z^{\star}\right\|=0$ with probability 1 for all $j$. However, we have shown that the sequence $\sum_{i=1}^{m}\left\|z_{k}^{i}-z^{*}\right\|$ converges with probability 1 for any $z^{*} \in X^{*} \times\left\{\eta^{*}\right\}$, which in view of $z^{\star} \in X^{*} \times\left\{\eta^{*}\right\}$, implies that $\lim _{k \rightarrow \infty}\left\|z_{k}^{j}-z^{\star}\right\|=0$ with probability 1 for all $j$. This is equivalent to $x_{k}^{j} \rightarrow x^{\star}$ and $\eta_{k}^{j} \rightarrow \eta^{*}$ with probability 1 for all $j$. 
Lemma 4 is critical for Theorem 2. In turn, the adjustment of the min-max estimates in $v_{k}^{i}$ of (10) is critical for validity of the lemma and, thus, for the convergence of the algorithm.

\section{EXAMPLE: UPLINK POWER CONTROL}

In this section we show the suitability of our algorithm to achieve a min-max fair allocation of utility in a cellular network. Due to the space limitation we will keep our discussion brief and refer the readers to [13] for a general discussion on the power allocation problem. We will be using the formulation discussed in [14]. There are $m$ mobile users (MU) in neighboring cells communicating with their respective base stations (BS) using a common wireless channel. Let $p_{i}$ denote the power used by MU $i$ to communicate with its base station. Due to the shared nature of the wireless medium the total received SINR at BS $i$ is given by

$$
\gamma_{i}\left(\bar{p} \in \Pi, \bar{h}_{i}\right)=\frac{p_{i} h_{i, i}^{2}}{\sigma_{i}^{2}+\sum_{j \neq i} p_{j} h_{i, j}^{2}},
$$

where $h_{i, j}$ is the channel coefficient between MU $j$ and BS $i$, and $\sigma_{i}^{2}$ is the receiver noise variance. The vector containing power variables $p_{i}$ is denoted $\bar{p}$ and the vector of channel coefficients at BS $i$ is denoted $\bar{h}_{i}$. The power variables are constrained to a maximum value of $p_{t}$, i.e., $p_{i} \leq p_{t}$ for all $i$.

Let $U_{i}\left(\gamma_{i}\left(\bar{p}, \bar{h}_{i}\right)\right)$ be the utility derived by BS $i$ and $V\left(p_{i}\right)$ be a cost function penalizing excessive power. Then, we are interested in finding an allocation that minimizes the worst case loss to any agent $i$. In other words, we wish to solve the following problem:

$$
\min _{\bar{p} \in \Pi} \max _{i \in V} \sum_{i=1}^{m}\left[V\left(p_{i}\right)-U_{i}\left(\gamma_{i}\left(\bar{p}, \bar{h}_{i}\right)\right)\right],
$$

where $\Pi=\left\{\bar{p} \in \mathbb{R}^{m} \mid p_{i} \leq p_{t}\right.$ for all $\left.i\right\}$ and $p_{t}$ is the maximum power. We consider the logarithmic utility function $U_{i}(x)=\log (x)$. In this case, using the transformation $p_{i}=e^{x_{i}}$, it can be shown that the preceding problem can be cast in the form of (1) with

$f_{i}(x)=\log \left(\sigma_{i}^{2} h_{i, i}^{-2} e^{-x_{i}}+\sum_{j \neq i} h_{i, i}^{-2} h_{j, i}^{2} e^{x_{j}-x_{i}}\right)+V\left(e^{x_{i}}\right)$,

and $X=\left\{x \mid x_{i} \leq \log \left(p_{t}\right)\right.$ for all $\left.i\right\}$.

In our simulations, we considered a cellular network of 16 square cells of the same size. Within each cell, the MU is randomly located and the base station is located at the center of the cell. The network under consideration is shown in Figure 1 . The channel coefficient $h_{i, j}$ is assumed to decay as the fourth power of the distance between the MU $j$ and the BS $i$. The shadow fading is assumed to be log-normal with variance 0.1 . The receiver noise variance $\sigma_{i}^{2}$ is taken to be 0.01 . The cost of the power is modeled as $V\left(p_{i}\right)=10^{-3} p_{i}$. The

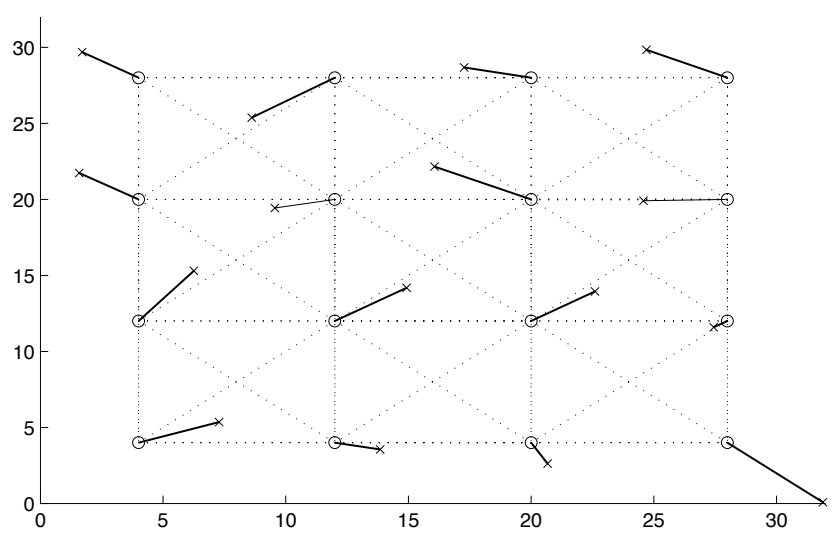

Fig. 1. The circles denote the base stations. The dotted lines denote the communication links between adjacent BSs. The cross denotes the MUs. The bold lines connect each MU to its respective $\mathrm{BS}$.

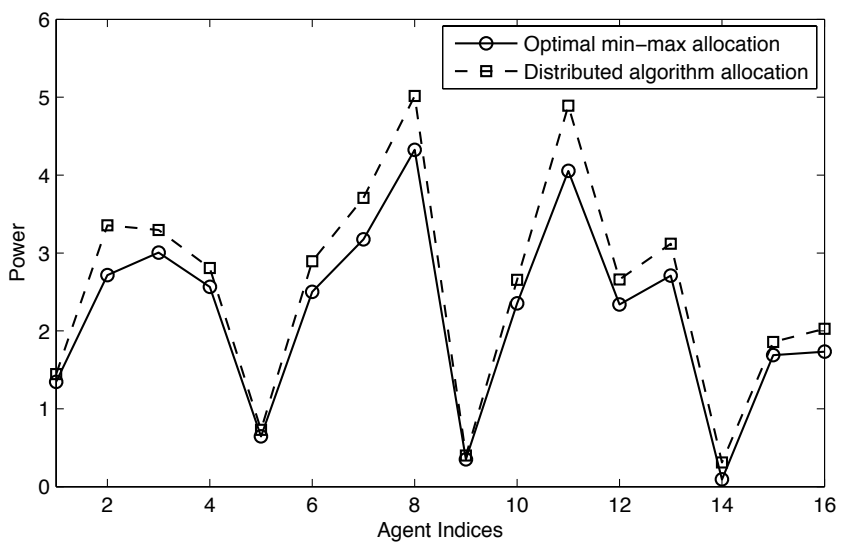

Fig. 2. The final iterate values after 2000 iterations of the algorithm.

step size is taken to be $\alpha_{k}=\frac{50}{t^{0.65}}$. The parameter $r_{i}$ in the algorithm (10) is chosen to be 1.3 for all $i$. Figure 2 shows the behavior of the algorithm (9)-(10) for this problem. The optimal min-max allocation was computed using a centralized gradient descent scheme for the penalized problem (4).

\section{REFERENCES}

[1] J.N. Tsitsiklis and M. Athans, "Convergence and asymptotic agreement in distributed decision problems," IEEE Transactions on Automatic Control, vol. 29, pp. 42-50, 1984.

[2] S. Kar and J.M.F. Moura, "Distributed consensus algorithms in sensor networks with imperfect communication: link failures and channel noise," IEEE Transac- 
tions on Signal Processing, vol. 57, no. 1, pp. 355-369, 2009.

[3] S.S. Stanković, M.S. Stanković, and D.M. Stipanović, "Decentralized parameter estimation by consensus based stochastic approximation," in Decision and Control, 2007 46th IEEE Conference on, dec. 2007, pp. 1535 -1540 .

[4] S.S. Ram, A. Nedić, and V.V. Veeravalli, "Distributed stochastic subgradient projection algorithms for convex optimization," Journal of Optimization Theory and Applications, vol. 147, no. 3, pp. 516-545, 2010.

[5] H. Boche, M. Wiczanowski, and S. Stanczak, "Unifying view on min-max fairness and utility optimization in cellular networks," in IEEE Wireless Communications and Networking Conference, 2005, vol. 3, pp. $1280-$ 1285.

[6] D.L. Donoho, "For most large underdetermined systems of linear equations the minimal $l 1$-norm solution is also the sparsest solution," Comm. Pure Appl. Math, vol. 59, pp. 797-829, 2004.

[7] V.S. Borkar, Stochastic Approximation: A Dynamical Systems Viewpoint, Cambridge University Press, 2008.

[8] H. Kushner and G. Yin, Stochastic Approximation and Recursive Algorithms and Applications, Springer, 2003.

[9] Y. Ermoliev, "Stochastic quazigradient methods," in Numerical Techniques for Stochastic Optimization, pp. 141-186. Springer-Verlag, N.Y., 1988.

[10] D.P. Bertsekas, A. Nedić, and A. Ozdaglar, Convex Analysis and Optimization, Athena Scientific, Belmont, MA, USA, 2003.

[11] D.P. Bertsekas, "Necessary and sufficient conditions for a penalty method to be exact," Mathematical Programming, vol. 9, pp. 87-99, 1975.

[12] B.T. Polyak, Introduction to Optimization, Optimization Software, Inc., New York, 1987.

[13] M. Chiang, P. Hande, T. Lan, and C.W. Tan, "Power Control in Wireless Cellular Networks," Found. Trends Netw., vol. 2, no. 4, pp. 381-533, 2008.

[14] S. S. Ram, V. V. Veeravalli, and A. Nedić, "Distributed non-autonomous power control through distributed convex optimization," in IEEE INFOCOM, 2009, pp. 30013005 . 